\title{
Mixed monotone-generalized contractions in partially ordered probabilistic metric spaces
}

\author{
Ljubomir Ćirić $^{1}$, Ravi P Agarwal ${ }^{2^{*}}$ and Bessem Samet ${ }^{3}$
}

\author{
* Correspondence: agarwal@fit.edu \\ ${ }^{2}$ Department of Mathematical \\ Sciences, Florida Institute of \\ Technology, Melbourne, FL 32901, \\ USA \\ Full list of author information is \\ available at the end of the article
}

\begin{abstract}
In this article, a new concept of mixed monotone-generalized contraction in partially ordered probabilistic metric spaces is introduced, and some coupled coincidence and coupled fixed point theorems are proved. The theorems Presented are an extension of many existing results in the literature and include several recent developments.

Mathematics Subject Classification: Primary 54H25; Secondary 47H10.

Keywords: probabilistic metric spacemixed monotone property, partially ordered set, coupled coincidence fixed point, coupled fixed point
\end{abstract}

\section{Introduction}

The Banach contraction principle [1] is one of the most celebrated fixed point theorem. Many generalizations of this famous theorem and other important fixed point theorems exist in the literature (cf. [2-37]).

Ran and Reurings [3] proved the Banach contraction principle in partially ordered metric spaces. Recently Agarwal et al. [2] presented some new fixed point results for monotone and generalized contractive type mappings in partially ordered metric spaces. Bhaskar and Lakshmikantham [4] initiated and proved some new coupled fixed point results for mixed monotone and contraction mappings in partially ordered metric spaces. The main idea in [2-11] involve combining the ideas of iterative technique in the contraction mapping principle with those in the monotone technique.

In [3], Ran and Reurings proved the following Banach type principle in partially ordered metric spaces.

Theorem 1 (Ran and Reurings [3]). Let $(X, \leq)$ be a partially ordered set such that every pair $x, y \in X$ has a lower and an upper bound. Let $d$ be a metric on $X$ such that the metric space $(X, d)$ is complete. Let $f: X \rightarrow X$ be a continuous and monotone (that is, either decreasing or increasing) operator. Suppose that the following two assertions hold:

(1) there exists $k \in(0,1)$ such that $d(f(x), f(y)) \leq k d(x, y)$, for each $x, y \in X$ with $x \geq y$,

(2) there exists $x_{0} \in X$ such that $x_{0} \leq f\left(x_{0}\right)$ or $x_{0} \geq f\left(x_{0}\right)$.

Then $f$ has a unique fixed point $x^{*} \in X$, i.e. $f\left(x^{*}\right)=x^{*}$, and for each $x \in X$, the sequence $\left\{f^{n}(x)\right\}$ of successive approximations of $f$ starting from $x$ converges to $x^{*} \in X$.

(C) 2011 Ćirićć et al; licensee Springer. This is an Open Access article distributed under the terms of the Creative Commons Attribution License (http://creativecommons.org/licenses/by/2.0), which permits unrestricted use, distribution, and reproduction in any medium, provided the original work is properly cited. 
The results of Ran and Reurings [3] have motivated many authors to undertake further investigation of fixed points in the field of ordered metric spaces: Agarwal et al. [2], Bhaskar and Lakshmikantham [4], Bhaskar et al. [5], Ćirić and Lakshmikantham [7], Ćirić et al. [8,9], Lakshmikantham and Ćirić [10], Nieto and López [6,11], Samet [12-14], and others.

Fixed point theory in probabilistic metric spaces can be considered as a part of probabilistic analysis, which is a very dynamic area of mathematical research. The theory of probabilistic metric spaces was introduced in 1942 by Menger [15]. These are generalizations of metric spaces in which the distances between points are described by probability distributions rather than by numbers. Schweizer and Sklar $[16,17]$ studied this concept and gave some fundamental results on these spaces. In 1972, Sehgal and Bharucha-Reid [18] initiated the study of contraction mappings on probabilistic metric spaces. Since then, several results have been obtained by various authors in this direction. For more details, we refer the reader to [19-27].

In [8], Ćirić et al. introduced the concept of monotone generalized contraction in partially ordered probabilistic metric spaces and proved some fixed and common fixed point theorems on such spaces.

In this article, we introduce a new concept of mixed monotone generalized contraction in partially ordered probabilistic metric spaces and we prove some coupled coincidence and coupled fixed point theorems on such spaces. Presented theorems extend many existing results in the literature, in particular, the results obtained by Bhaskar and Lakshmikantham [4], Lakshmikantham and Ćirić [10], and include several recent developments.

Throughout this article, the space of all probability distribution functions is denoted by $\Delta^{+}=\{F: \mathbb{R} \cup\{-\infty,+\infty\} \rightarrow[0,1]: F$ is left-continuous and non-decreasing on $\mathbb{R}, F(0)$ $=0$ and $F(+\infty)=1\}$ and the subset $D^{+} \subseteq \Delta^{+}$is the set $D^{+}=\left\{F \in \Delta^{+}: \lim _{t \rightarrow+\infty} F(t)=\right.$ $1\}$. The space $\Delta^{+}$is partially ordered by the usual point-wise ordering of functions, i.e., $F \leq G$ if and only if $F(t) \leq G(t)$ for all $t$ in $\mathbb{R}$. The maximal element for $\Delta^{+}$in this order is the distribution function given by

$$
\varepsilon_{0}(t)= \begin{cases}0, & \text { if } t \leq 0 \\ 1, & \text { if } t>0\end{cases}
$$

We refer the reader to [22] for the terminology concerning probabilistic metric spaces (also called Menger spaces).

\section{Main results}

We start by recalling some definitions introduced by Bhaskar and Lakshmikantham [4] and Lakshmikantham and Ćirić [10].

Definition 2 (Bhaskar and Lakshmikantham [4]). Let $X$ be a non-empty set and $A$ : $X \times X \rightarrow X$ be a given mapping. An element $(x, y) \in X \times X$ is said to be a coupled fixed point of $A$ if

$$
A(x, y)=x \text { and } A(y, x)=y .
$$

Definition 3 (Lakshmikantham and Ćirić [10]). Let $X$ be a non-empty set, $A: X \times$ $X \rightarrow X$ and $h: X \rightarrow X$ are given mappings.

(1) An element $(x, y) \in X \times X$ is said to be a coupled coincidence point of $A$ and $h$ if

$$
A(x, y)=h(x) \text { and } A(y, x)=h(y) .
$$


(2) An element $(x, y) \in X \times X$ is said to be a coupled common fixed point of $A$ and $h$ if $A(x, y)=h(x)=x \quad$ and $\quad A(y, x)=h(y)=y$.

(3) We say that $A$ and $h$ commute at $(x, y) \in X \times X$ if

$$
h(A(x, y))=A(h(x), h(y)) \quad \text { and } \quad h(A(y, x))=A(h(y), h(x))
$$

(4) A and h commute if

$$
h(A(x, y))=A(h(x), h(y)), \quad \text { for all }(x, y) \in X \times X .
$$

Definition 4 (Lakshmikantham and Ćirić [10]). Let $(X, \leq)$ be a partially ordered set, $A: X \times X \rightarrow X$ and $h: X \rightarrow X$ are given mappings. We say that $A$ has the mixed $h$ monotone property if for all $x, y \in X$, we have

$$
\begin{array}{lll}
x_{1}, x_{2} & \in \quad X, \quad h\left(x_{1}\right) \leq h\left(x_{2}\right) \Rightarrow A\left(x_{1}, y\right) \leq A\left(x_{2}, y\right), \\
y_{1}, y_{2} & \in \quad X, \quad h\left(y_{1}\right) \geq h\left(y_{2}\right) \Rightarrow A\left(x, y_{1}\right) \leq A\left(x, y_{2}\right) .
\end{array}
$$

If $h$ is the identity mapping on $X$, then A satisfies the mixed monotone property.

We need the following lemmas to prove our main results.

Lemma 5. Let $n \geq 1$. If $F \in D^{+}, G_{1}, G_{2}, \ldots, G_{n}: \mathbb{R} \rightarrow[0,1]$ are non-decreasing functions and, for some $k \in(0,1)$,

$$
F(k t) \geq \min \left\{G_{1}(t), G_{2}(t), \cdots, G_{n}(t), F(t)\right\}, \forall t>0,
$$

then $F(k t) \geq \min \left\{G_{1}(t), G_{2}(t), \ldots, G_{n}(t)\right\}$ for all $t>0$.

Proof. The proof is a simple adaptation of that of Lemma 3.3 in [8]. $\square$

Lemma 6. Let $(X, F, \Delta)$ be a Menger PM-space and $k \in(0,1)$. If

$$
\min \left\{F_{p, q}(k t), F_{s, v}(k t)\right\}=\min \left\{F_{p, q}(t), F_{s, v}(t)\right\}, \quad \text { for all } t>0,
$$

then $p=q$ and $s=v$.

Proof. From (2) it is easy to show by induction that

$$
\min \left\{F_{p, q}\left(k^{n} t\right), F_{s, v}\left(k^{n} t\right)\right\}=\min \left\{F_{p, q}(t), F_{s, v}(t)\right\}, \quad \text { for all } n \geq 1 .
$$

Now we shall show that $\min \left\{F_{p q}(t), F_{s, v}(t)\right\}=1$ for all $t>0$. Suppose, to the contrary, that there exists some $t_{0}>0$ such that $\min \left\{F_{p q}\left(t_{0}\right), F_{s, v}\left(t_{0}\right)\right\}<1$. Since $(X, F)$ is a Menger PM space, then $\min \left\{F_{p q}(t), F_{s, v}(t)\right\} \rightarrow 1$ as $t \rightarrow \infty$. Therefore, there exists $t_{1}>t_{0}$ such that

$$
\min \left\{F_{p q}\left(t_{1}\right), F_{s, v}\left(t_{1}\right)\right\}>\min \left\{F_{p q}\left(t_{0}\right), F_{s, v}\left(t_{0}\right)\right\}
$$

Since $t_{0}>0$ and $k \in(0,1)$, there exists a positive integer $n>1$ such that $k^{n} t_{1}<t_{0}$. Then by the monotony of $F_{p q}(\cdot)$ and $F_{s, v}(\cdot)$, it follows that $\min \left\{F_{p q}\left(k^{n} t_{1}\right), F_{s, v}\left(k^{n} t_{1}\right)\right\} \leq$ $\min \left\{F_{p q}\left(t_{0}\right), F_{s, v}\left(t_{0}\right)\right\}$. Hence and from (3) with $t=t_{1}$, we have

$$
\min \left\{F_{p q}\left(t_{1}\right), F_{s, v}\left(t_{1}\right)\right\}=\min \left\{F_{p q}\left(k^{n} t_{1}\right), F_{s, v}\left(k^{n} t_{1}\right)\right\} \leq \min \left\{F_{p q}\left(t_{0}\right), F_{s, v}\left(t_{0}\right\},\right.
$$

a contradiction with (4). Therefore $\min \left\{F_{p q}(t), F_{s, v}(t)\right\}=1$ for all $t>0$, which implies that $F_{p q}(t)=1$ and $F_{s, v}(t)=1$ for all $t>0$. Hence $p=q$ and $s=v$. $\square$

Now, we state and prove our first result. 
Theorem 7. Let $(X, \leq)$ be a partially ordered set and $(X, F, \Delta)$ be a complete Menger PM-space under a T-norm $\Delta$ of H-type (Hadžić-type). Suppose $A: X \times X \rightarrow X$ and $h$ : $X \rightarrow X$ are two mappings such that $A$ has the h-mixed monotone property on $X$ and, for some $k \in(0,1)$,

$$
\begin{aligned}
F_{A(x, y), A(u, v)}(k t) \geq & \min \left\{F_{h(x), h(u)}(t), F_{h(y), h(v)}(t), F_{h(x), A(x, y)}(t),\right. \\
& \left.F_{h(u), A(u, v)}(t), F_{h(y), A(\gamma, x)}(t), F_{h(v), A(v, u)}(t)\right\}
\end{aligned}
$$

for all $x, y \in X$ for which $h(x) \leq h(u)$ and $h(y) \geq h(v)$ and all $t>0$. Suppose also that $A(X \times X) \subseteq h(X), h(X)$ is closed and

if $\left\{h\left(x_{n}\right)\right\} \subset X$ is a non - decreasing sequence with $h\left(x_{n}\right) \rightarrow h(z)$ in $h(X)$

then $h\left(x_{n}\right) \leq h(z)$ for all $n$ hold,

if $\left\{h\left(x_{n}\right)\right\} \subset X$ is a non - decreasing sequence with $h\left(x_{n}\right) \rightarrow h(z)$ in $h(X)$

then $h(z) \leq h\left(x_{n}\right)$ for all $n$ hold.

If there exist $x_{0}, y_{0} \in X$ such that

$$
h\left(x_{0}\right) \leq A\left(x_{0}, y_{0}\right) \text { and } h\left(y_{0}\right) \geq A\left(y_{0}, x_{0}\right),
$$

then $A$ and $h$ have a coupled coincidence point, that is, there exist $p, q \in X$ such that $A(p, q)=h(p)$ and $A(q, p)=h(q)$.

Proof. By hypothesis, there exist $\left(x_{0}, y_{0}\right) \in X \times X$ such that $h\left(x_{0}\right) \leq A\left(x_{0}, y_{0}\right)$ and $h$ $\left(y_{0}\right) \geq A\left(y_{0}, x_{0}\right)$. Since $A(X \times X) \subseteq h(X)$, we can choose $x_{1}, y_{1} \in X$ such that $h\left(x_{1}\right)=A$ $\left(x_{0}, y_{0}\right)$ and $h\left(y_{1}\right)=A\left(y_{0}, x_{0}\right)$. Now $A\left(x_{1}, y_{1}\right)$ and $A\left(y_{1}, x_{1}\right)$ are well defined. Again, from $A(X \times X) \subseteq h(X)$, we can choose $x_{2}, y_{2} \in X$ such that $h\left(x_{2}\right)=A\left(x_{1}, y_{1}\right)$ and $h\left(y_{2}\right)=A$ $\left(y_{1}, x_{1}\right)$. Continuing this process, we can construct sequences $\left\{x_{n}\right\}$ and $\left\{y_{n}\right\}$ in $X$ such that

$$
h\left(x_{n+1}\right)=A\left(x_{n}, y_{n}\right) \text { and } h\left(y_{n+1}\right)=A\left(y_{n}, x_{n}\right), \text { for all } n \in \mathbb{N} .
$$

We shall show that

$$
h\left(x_{n}\right) \leq h\left(x_{n+1}\right), \quad \text { for all } n \in \mathbb{N}
$$

and

$$
h\left(y_{n}\right) \geq h\left(y_{n+1}\right), \quad \text { for all } n \in \mathbb{N} .
$$

We shall use the mathematical induction. Let $n=0$. Since $h\left(x_{0}\right) \leq A\left(x_{0}, y_{0}\right)$ and $h\left(y_{0}\right)$ $\geq A\left(y_{0}, x_{0}\right)$, and as $h\left(x_{1}\right)=A\left(x_{0}, y_{0}\right)$ and $h\left(y_{1}\right)=A\left(y_{0}, x_{0}\right)$, we have $h\left(x_{0}\right) \leq h\left(x_{1}\right)$ and $h$ $\left(y_{0}\right) \geq h\left(y_{1}\right)$. Thus (9) and (10) hold for $n=0$. Suppose now that (9) and (10) hold for some fixed $n \in \mathbb{N}$. Then, since $h\left(x_{n}\right) \leq h\left(x_{n+1}\right)$ and $h\left(y_{n+1}\right) \leq h\left(y_{n}\right)$, and as $A$ has the $h$ mixed monotone property, from (8),

$$
h\left(x_{n+1}\right)=A\left(x_{n}, y_{n}\right) \leq A\left(x_{n+1}, y_{n}\right) \quad \text { and } \quad A\left(y_{n+1}, x_{n}\right) \leq A\left(y_{n}, x_{n}\right)=h\left(y_{n+1}\right),
$$

and from (8),

$$
h\left(x_{n+2}\right)=A\left(x_{n+1}, y_{n+1}\right) \geq A\left(x_{n+1}, y_{n}\right) \text { and } A\left(y_{n+1}, x_{n}\right) \geq A\left(y_{n+1}, x_{n+1}\right)=h\left(y_{n+2}\right) .
$$

Now from (11) and (12), we get

$$
h\left(x_{n+1}\right) \leq h\left(x_{n+2}\right)
$$


and

$$
h\left(y_{n+1}\right) \geq h\left(y_{n+2}\right)
$$

Thus by the mathematical induction we conclude that (9) and (10) hold for all $n \in$ $\mathbb{N}$. Therefore,

$$
h\left(x_{0}\right) \leq h\left(x_{1}\right) \leq h\left(x_{2}\right) \leq h\left(x_{3}\right) \leq \cdots \leq h\left(x_{n}\right) \leq h\left(x_{n+1}\right) \leq \cdots
$$

and

$$
h\left(y_{0}\right) \geq h\left(y_{1}\right) \geq h\left(y_{2}\right) \geq h\left(y_{3}\right) \geq \cdots \geq h\left(y_{n}\right) \geq h\left(y_{n+1}\right) \geq \cdots .
$$

Now, from (13) and (14), we can apply (5) for $(x, y)=\left(x_{n}, y_{n}\right)$ and $(u, v)=\left(x_{n+1}, y_{n}\right.$ $\left.{ }_{+1}\right)$. Thus, for all $t>0$, we have

$$
\begin{aligned}
F_{A\left(x_{n}, y_{n}\right), A\left(x_{n+1}, y_{n+1}\right)}(k t) \geq & \min \left\{F_{h\left(x_{n}\right), h\left(x_{n+1}\right)}(t), F_{h\left(y_{n}\right), h\left(y_{n+1}\right)}(t), F_{h\left(x_{n}\right), A\left(x_{n}, y_{n}\right)}(t),\right. \\
& \left.F_{h\left(x_{n+1}\right), A\left(x_{n+1}, y_{n+1}\right)}(t), F_{h\left(y_{n}\right), A\left(y_{n}, x_{n}\right)}(t), F_{h\left(y_{n+1}\right), A\left(y_{n+1}, x_{n+1}\right)}(t)\right\} .
\end{aligned}
$$

Using (8), we obtain

$$
\begin{aligned}
F_{h\left(x_{n+1}\right), h\left(x_{n+2}\right)}(k t) \geq & \min \left\{F_{h\left(x_{n}\right), h\left(x_{n+1}\right)}(t), F_{h\left(y_{n}\right), h\left(y_{n+1}\right)}(t), F_{h\left(x_{n+1}\right), h\left(x_{n+2}\right)}(t),\right. \\
& \left.F_{h\left(y_{n+1}\right), h\left(y_{n+2}\right)}(t)\right\} .
\end{aligned}
$$

Similarly, from (13) and (14), we can apply (5) for $(x, y)=\left(y_{n+1}, x_{n+1}\right)$ and $(u, v)=$ $\left(y_{n}, x_{n}\right)$. Thus, by using (8), for all $t>0$ we get

$$
\begin{aligned}
F_{h\left(y_{n+2}\right), h\left(y_{n+1}\right)}(k t) \geq & \min \left\{F_{h\left(y_{n+1}\right), h\left(y_{n}\right)}(t), F_{h\left(x_{n+1}\right), h\left(x_{n}\right)}(t), F_{h\left(y_{n+1}\right), h\left(y_{n+2}\right)}(t),\right. \\
& \left.F_{h\left(x_{n+1}\right), h\left(x_{n+2}\right)}(t)\right\} .
\end{aligned}
$$

From (15) and (16), we have

$$
\begin{aligned}
& \min \left\{F_{h\left(x_{n+1}\right), h\left(x_{n+2}\right)}(k t), F_{h\left(y_{n+1}\right), h\left(y_{n+2}\right)}(k t)\right\} \\
\geq & \min \left\{F_{h\left(x_{n}\right), h\left(x_{n+1}\right)}(t), F_{h\left(y_{n}\right), h\left(y_{n+1}\right)}(t), F_{h\left(x_{n+1}\right), h\left(x_{n+2}\right)}(t), F_{h\left(y_{n+1}\right), h\left(y_{n+2}\right)}(t)\right\} \\
= & \min \left\{F_{h\left(x_{n}\right), h\left(x_{n+1}\right)}(t), F_{h\left(y_{n}\right), h\left(y_{n+1}\right)}(t), \min \left\{F_{h\left(x_{n+1}\right), h\left(x_{n+2}\right)}(t), F_{h\left(y_{n+1}\right), h\left(y_{n+2}\right)}(t)\right\}\right\} .
\end{aligned}
$$

Now, from Lemma 5, we have

$$
\min \left\{F_{h\left(x_{n+1}\right), h\left(x_{n+2}\right)}(k t), F_{h\left(y_{n+1}\right), h\left(y_{n+2}\right)}(k t)\right\} \geq \min \left\{F_{h\left(x_{n}\right), h\left(x_{n+1}\right)}(t), F_{h\left(y_{n}\right), h\left(y_{n+1}\right)}(t)\right\}
$$

for all $t>0$. From (17) it follows that

$$
\min \left\{F_{h\left(x_{n+1}\right), h\left(x_{n+2}\right)}(t), F_{h\left(y_{n+1}\right), h\left(y_{n+2}\right)}(t)\right\} \geq \min \left\{F_{h\left(x_{n}\right), h\left(x_{n+1}\right)}(t / k), F_{h\left(y_{n}\right), h\left(y_{n+1}\right)}(t / k)\right\}
$$

for all $t>0$. Repeating the inequality (18), for all $t>0$ we get

$$
\begin{aligned}
\min \left\{F_{h\left(x_{n+1}\right), h\left(x_{n+2}\right)}(t), F_{h\left(y_{n+1}\right), h\left(y_{n+2}\right)}(t)\right\} & \geq \min \left\{F_{h\left(x_{n}\right), h\left(x_{n+1}\right)}(t / k), F_{h\left(y_{n}\right), h\left(y_{n+1}\right)}(t / k)\right\} \\
& \geq \min \left\{F_{h\left(x_{n-1}\right), h\left(x_{n}\right)}\left(t / k^{2}\right), F_{h\left(y_{n-1}\right), h\left(y_{n}\right)}\left(t / k^{2}\right)\right\} \\
& \geq \cdots \\
& \geq \min \left\{F_{h\left(x_{0}\right), h\left(x_{1}\right)}\left(t / k^{n+1}\right), F_{h\left(y_{0}\right), h\left(y_{1}\right)}\left(t / k^{n+1}\right)\right\} .
\end{aligned}
$$

Thus

$$
\min \left\{F_{h\left(x_{n+1}\right), h\left(x_{n+2}\right)}(t), F_{h\left(y_{n+1}\right), h\left(y_{n+2}\right)}(t)\right\} \geq \min \left\{F_{h\left(x_{0}\right), h\left(x_{1}\right)}\left(t / k^{n+1}\right), F_{h\left(y_{0}\right), h\left(y_{1}\right)}\left(t / k^{n+1}\right)\right\},
$$


for all $t>0$ and $n \in \mathbb{N}$. Letting $n \rightarrow+\infty$ in (19), we obtain

$$
\lim _{n \rightarrow \infty} F_{h\left(x_{n}\right), h\left(x_{n+1}\right)}(t)=1, \quad \text { for all } t>0,
$$

and

$$
\lim _{n \rightarrow \infty} F_{h\left(y_{n}\right), h\left(y_{n+1}\right)}(t)=1, \quad \text { for all } t>0 .
$$

We now prove that $\left\{h\left(x_{n}\right)\right\}$ and $\left\{h\left(y_{n}\right)\right\}$ are Cauchy sequences in $X$. We need to show that for each $\delta>0$ and $0<\varepsilon<1$, there exists a positive integer $n_{0}=n_{0}(\delta, \varepsilon)$ such that

$$
F_{h\left(x_{n}\right), h\left(x_{m}\right)}(\delta)>1-\varepsilon, \quad \text { for all } m>n \geq n_{0}(\delta, \varepsilon)
$$

and

$$
F_{h\left(y_{n}\right), h\left(y_{m}\right)}(\delta)>1-\varepsilon, \quad \text { for all } m>n \geq n_{0}(\delta, \varepsilon),
$$

that is,

$$
\min \left\{F_{h\left(x_{n}\right), h\left(x_{m}\right)}(\delta), F_{h\left(y_{n}\right), h\left(y_{m}\right)}(\delta)\right\}>1-\varepsilon, \quad \text { for all } m>n \geq n_{0}(\delta, \varepsilon) .
$$

Now we shall prove that for each $\rho>0$,

$$
\begin{aligned}
& \min \left\{F_{h\left(x_{n}\right), h\left(x_{m}\right)}(\rho), F_{h\left(y_{n}\right), h\left(y_{m}\right)}(\rho)\right\} \\
\geq & \Delta^{m-n}\left(\min \left\{F_{h\left(x_{n}\right), h\left(x_{n+1}\right)}(\rho-k \rho), F_{h\left(y_{n}\right), h\left(y_{n+1}\right)}(\rho-k \rho)\right\}\right)
\end{aligned}
$$

for all $m \geq n+1$. We prove (23) by the mathematical induction. Let $m=n+1$. Then from monotony of $F$, for $m=n+1$ we have

$$
\begin{aligned}
F_{h\left(x_{n}\right), h\left(x_{n+1}\right)}(\rho) & \geq F_{h\left(x_{n}\right), h\left(x_{n+1}\right)}(\rho-k \rho) \\
& =\Delta\left(F_{h\left(x_{n}\right), h\left(x_{n+1}\right)}(\rho-k \rho), 1\right) \\
& \geq \Delta\left(F_{h\left(x_{n}\right), h\left(x_{n+1}\right)}(\rho-k \rho), F_{h\left(x_{n}\right), h\left(x_{n+1}\right)}(\rho-k \rho)\right) \\
& =\Delta^{1}\left(F_{h\left(x_{n}\right), h\left(x_{n+1}\right)}(\rho-k \delta)\right) \\
& \geq \Delta^{1}\left(\min \left\{F_{h\left(x_{n}\right), h\left(x_{n+1}\right)}(\rho-k \rho), F_{h\left(y_{n}\right), h\left(y_{n+1}\right)}(\rho-k \rho)\right\}\right) .
\end{aligned}
$$

Similarly,

$$
\begin{aligned}
F_{h\left(y_{n}\right), h\left(y_{n+1}\right)}(\rho) & \geq F_{h\left(y_{n}\right), h\left(y_{n+1}\right)}(\rho-k \rho) \\
& =\Delta\left(F_{h\left(y_{n}\right), h\left(y_{n+1}\right)}(\rho-k \rho), 1\right) \\
& \geq \Delta\left(F_{h\left(y_{n}\right), h\left(y_{n+1}\right)}(\rho-k \rho), F_{h\left(y_{n}\right), h\left(y_{n+1}\right)}(\rho-k \rho)\right) \\
& =\Delta^{1}\left(F_{h\left(y_{n}\right), h\left(y_{n+1}\right)}(\rho-k \delta)\right) \\
& \geq \Delta^{1}\left(\min \left\{F_{h\left(x_{n}\right), h\left(x_{n+1}\right)}(\rho-k \rho), F_{h\left(y_{n}\right), h\left(y_{n+1}\right)}(\rho-k \rho)\right\}\right) .
\end{aligned}
$$

Then

$$
\min \left\{F_{h\left(x_{n}\right), h\left(x_{n+1}\right)}(\rho), F_{h\left(y_{n}\right), h\left(y_{n+1}\right)}(\rho)\right\} \geq \Delta^{1}\left(\min \left\{F_{h\left(x_{n}\right), h\left(x_{n+1}\right)}(\rho-k \rho), F_{h\left(y_{n}\right), h\left(y_{n+1}\right)}(\rho-k \rho)\right\}\right),
$$

and (23) holds for $m=n+1$.

Suppose now that (23) holds for some $m \geq n+1$. Since $\rho-k \rho>0$, from the probabilistic triangle inequality, we have

$$
\begin{aligned}
F_{h\left(x_{n}\right), h\left(x_{m+1}\right)}(\rho) & =F_{h\left(x_{n}\right), h\left(x_{m+1}\right)}((\rho-k \rho)+k \rho) \\
& \geq \Delta\left(F_{h\left(x_{n}\right), h\left(x_{n+1}\right)}(\rho-k \rho), F_{h\left(x_{n+1}\right), h\left(x_{m+1}\right)}(k \rho)\right) .
\end{aligned}
$$

Similarly,

$$
F_{h\left(y_{n}\right), h\left(y_{m+1}\right)}(\rho) \geq \Delta\left(F_{h\left(y_{n}\right), h\left(y_{n+1}\right)}(\rho-k \rho), F_{h\left(y_{n+1}\right), h\left(y_{m+1}\right)}(k \rho)\right) .
$$


From (24) and (25), we get

$$
\begin{aligned}
& \min \left\{F_{h\left(x_{n}\right), h\left(x_{m+1}\right)}(\rho), F_{h\left(y_{n}\right), h\left(y_{m+1}\right)}(\rho)\right\} \\
& \geq \Delta\left(\min \left\{F_{h\left(x_{n}\right), h\left(x_{n+1}\right)}(\rho-k \rho), F_{h\left(y_{n}\right), h\left(y_{n+1}\right)}(\rho-k \rho)\right\}, \min \left\{F_{h\left(x_{n+1}\right), h\left(x_{m+1}\right)}(k \rho), F_{h\left(y_{n+1}\right), h\left(y_{m+1}\right)}(k \rho)\right\}\right) .
\end{aligned}
$$

Now we shall consider $\min \left\{F_{h\left(x_{n+1}\right), h\left(x_{m+1}\right)}(k \rho), F_{h\left(y_{n+1}\right), h\left(y_{m+1}\right)}(k \rho)\right\}$. From (5) and the hypothesis (23), we have

$$
\begin{aligned}
& \min \left\{F_{h\left(x_{n+1}\right), h\left(x_{m+1}\right)}(k \rho), F_{h\left(y_{n+1}\right), h\left(y_{m+1}\right)}(k \rho)\right\} \\
= & \min \left\{F_{A\left(x_{n}, y_{n}\right), A\left(x_{m}, y_{m}\right)}(k \rho), F_{A\left(y_{n}, x_{n}\right), A\left(y_{m}, x_{m}\right)}(k \rho)\right\} \\
\geq & \min \left\{F_{h\left(x_{n}\right), h\left(x_{m}\right)}(\rho), F_{h\left(y_{n}\right), h\left(y_{m}\right)}(\rho),\right. \\
& \left.F_{h\left(x_{n}\right), h\left(x_{n+1}\right)}(\rho), F_{h\left(x_{m}\right), h\left(x_{m+1}\right)}(\rho), F_{h\left(y_{n}\right), h\left(y_{n+1}\right)}(\rho), F_{h\left(y_{m}\right), h\left(y_{m+1}\right)}(\rho)\right\} \\
= & \min \left\{\min \left\{F_{h\left(x_{n}\right), h\left(x_{m}\right)}(\rho), F_{h\left(y_{n}\right), h\left(y_{m}\right)}(\rho)\right\}\right. \\
& \left.F_{h\left(x_{n}\right), h\left(x_{n+1}\right)}(\rho), F_{h\left(x_{m}\right), h\left(x_{m+1}\right)}(\rho), F_{h\left(y_{n}\right), h\left(y_{n+1}\right)}(\rho), F_{h\left(y_{m}\right), h\left(y_{m+1}\right)}(\rho)\right\} \\
\geq & \min \left\{\Delta^{m-n}\left(\min \left\{F_{h\left(x_{n}\right), h\left(x_{n+1}\right)}(\rho-k \rho), F_{h\left(y_{n}\right), h\left(y_{n+1}\right)}(\rho-k \rho)\right\}\right),\right. \\
& \left.F_{h\left(x_{n}\right), h\left(x_{n+1}\right)}(\rho), F_{h\left(x_{m}\right), h\left(x_{m+1}\right)}(\rho), F_{h\left(y_{n}\right), h\left(y_{n+1}\right)}(\rho), F_{h\left(y_{m}\right), h\left(y_{m+1}\right)}(\rho)\right\} .
\end{aligned}
$$

Note that from (18), for every positive integer $m \geq n$, we have

$$
\begin{aligned}
& \min \left\{F_{h\left(x_{m}\right), h\left(x_{m+1}\right)}(\rho), F_{h\left(y_{m}\right), h\left(y_{m+1}\right)}(\rho)\right\} \\
\geq & \min \left\{F_{h\left(x_{n}\right), h\left(x_{n+1}\right)}\left(\rho / k^{m-n}\right), F_{h\left(y_{n}\right), h\left(y_{n+1}\right)}\left(\rho / k^{m-n}\right)\right\} \\
\geq & \min \left\{F_{h\left(x_{n}\right), h\left(x_{n+1}\right)}(\rho), F_{h\left(y_{n}\right), h\left(y_{n+1}\right)}(\rho)\right\} \quad \text { for all } n \in \mathbb{N} .
\end{aligned}
$$

Therefore, from (27) and (28), we get

$$
\begin{aligned}
& \min \left\{F_{h\left(x_{n+1}\right), h\left(x_{m+1}\right)}(k \rho), F_{h\left(y_{n+1}\right), h\left(y_{m+1}\right)}(k \rho)\right\} \\
\geq & \min \left\{\Delta^{m-n}\left(\min \left\{F_{h\left(x_{n}\right), h\left(x_{n+1}\right)}(\rho-k \rho), F_{h\left(y_{n}\right), h\left(y_{n+1}\right)}(\rho-k \rho)\right\}\right),\right. \\
& \left.\min \left\{F_{h\left(x_{n}\right), h\left(x_{n+1}\right)}(\rho), F_{h\left(y_{n}\right), h\left(y_{n+1}\right)}(\rho)\right\}\right\} .
\end{aligned}
$$

Since $\rho \geq \rho-k \rho$, using the monotony of $F$, we have

$$
\min \left\{F_{h\left(x_{n}\right), h\left(x_{n+1}\right)}(\rho), F_{h\left(y_{n}\right), h\left(y_{n+1}\right)}(\rho)\right\} \geq \min \left\{F_{h\left(x_{n}\right), h\left(x_{n+1}\right)}(\rho-k \rho), F_{h\left(y_{n}\right), h\left(y_{n+1}\right)}(\rho-k \rho)\right\} .
$$

Then, we have

$$
\begin{aligned}
& \min \left\{F_{h\left(x_{n+1}\right), h\left(x_{m+1}\right)}(k \rho), F_{h\left(y_{n+1}\right), h\left(y_{m+1}\right)}(k \rho)\right\} \\
\geq & \min \left\{\Delta^{m-n}\left(\min \left\{F_{h\left(x_{n}\right), h\left(x_{n+1}\right)}(\rho-k \rho), F_{h\left(y_{n}\right), h\left(y_{n+1}\right)}(\rho-k \rho)\right\}\right),\right. \\
& \left.\min \left\{F_{h\left(x_{n}\right), h\left(x_{n+1}\right)}(\rho-k \rho), F_{h\left(y_{n}\right), h\left(y_{n+1}\right)}(\rho-k \rho)\right\}\right\} .
\end{aligned}
$$

Since $\left\{\Delta^{i}(t)\right\}_{i \geq 0}$ is a decreasing sequence for all $t>0$, we have

$$
\begin{aligned}
& \min \left\{F_{h\left(x_{n}\right), h\left(x_{n+1}\right)}(\rho-k \rho), F_{h\left(y_{n}\right), h\left(y_{n+1}\right)}(\rho-k \rho)\right\} \\
\geq & \Delta^{m-n}\left(\min \left\{F_{h\left(x_{n}\right), h\left(x_{n+1}\right)}(\rho-k \rho), F_{h\left(y_{n}\right), h\left(y_{n+1}\right)}(\rho-k \rho)\right\}\right) .
\end{aligned}
$$

Then, we get

$$
\begin{aligned}
& \min \left\{F_{h\left(x_{n+1}\right), h\left(x_{m+1}\right)}(k \rho), F_{h\left(y_{n+1}\right), h\left(y_{m+1}\right)}(k \rho)\right\} \\
\geq & \Delta^{m-n}\left(\min \left\{F_{h\left(x_{n}\right), h\left(x_{n+1}\right)}(\rho-k \rho), F_{h\left(y_{n}\right), h\left(y_{n+1}\right)}(\rho-k \rho)\right\}\right) .
\end{aligned}
$$

Now, from (26) and (29), we obtain

$$
\begin{aligned}
& \min \left\{F_{h\left(x_{n}\right), h\left(x_{m+1}\right)}(\rho), F_{h\left(y_{n}\right), h\left(y_{m+1}\right)}(\rho)\right\} \\
\geq & \Delta\left(\Delta^{m-n}\left(\min \left\{F_{h\left(x_{n}\right), h\left(x_{n+1}\right)}(\rho-k \rho), F_{h\left(y_{n}\right), h\left(y_{n+1}\right)}(\rho-k \rho)\right\}\right),\right. \\
& \left.\min \left\{F_{h\left(x_{n}\right), h\left(x_{n+1}\right)}(\rho-k \rho), F_{h\left(y_{n}\right), h\left(y_{n+1}\right)}(\rho-k \rho)\right\}\right) \\
= & \Delta^{m-n+1}\left(\min \left\{F_{h\left(x_{n}\right), h\left(x_{n+1}\right)}(\rho-k \rho), F_{h\left(y_{n}\right), h\left(y_{n+1}\right)}(\rho-k \rho)\right\}\right) .
\end{aligned}
$$


Hence and by the induction we conclude that (23) holds for all $m \geq n+1$.

Now we show that $\left\{h\left(x_{n}\right)\right\}$ and $\left\{h\left(y_{n}\right)\right\}$ are Cauchy sequences, that is, for each $\delta>0$ and $0<\varepsilon<1$, there exists a positive integer $n_{0}=n_{0}(\delta, \varepsilon)$ such that (22) holds. Since $\Delta$ is of $H$-type, then $\left\{\Delta^{n}: n \in+\right\}$ is equicontinuous at 1 , that is,

$$
\forall \varepsilon \in(0,1) \exists r \in(0,1) \mid s>1-r \Rightarrow \Delta^{n}(s)>1-\varepsilon(\text { for all } n \in \mathbb{N}) .
$$

Since $\delta-k \delta>0$, from (20) and (21) it follows that for any $0<r<1$ there exists a positive integer $n_{1}=n_{1}((\delta-k \delta), r)$ such that

$$
F_{h\left(x_{n}\right), h\left(x_{n+1}\right)}(\delta-k \delta)>1-r \quad \text { and } \quad F_{h\left(y_{n}\right), h\left(y_{n+1}\right)}(\delta-k \delta)>1-r, \quad \text { for all } \quad n \geq n_{1} .
$$

Then by (23), with $\min \left\{F_{h\left(x_{n}\right), h\left(x_{n+1}\right)}(\rho-k \rho), F_{h\left(y_{n}\right), h\left(y_{n+1}\right)}(\rho-k \rho)\right\}=s$, we conclude that (22) holds for $n_{0}(\delta, \varepsilon)=n_{1}((\delta-k \delta), r)$. Thus we proved that $\left\{h\left(x_{n}\right)\right\}$ and $\left\{h\left(y_{n}\right)\right\}$ are Cauchy sequences in $X$.

Since $h(X)$ is complete, there is some $p, q \in X$ such that

$$
\lim _{n \rightarrow \infty} h\left(x_{n}\right)=h(p) \text { and } \lim _{n \rightarrow \infty} h\left(y_{n}\right)=h(q),
$$

that is, for all $t>0$,

$$
\lim _{n \rightarrow \infty} F_{h\left(x_{n}\right), h(p)}(t)=1 \quad \text { and } \quad \lim _{n \rightarrow \infty} F_{h\left(y_{n}\right), h(q)}(t)=1 .
$$

Now we show that $(p, q)$ is a coupled coincidence point of $A$ and $h$.

Since $\left\{h\left(x_{n}\right)\right\}$ is a non-decreasing sequence, from (30) and (6), we have

$$
h\left(x_{n}\right) \leq h(p) .
$$

Since $\left\{h\left(y_{n}\right)\right\}$ is a non-increasing sequence, from (30) and (7), we have

$$
h(q) \leq h\left(y_{n}\right) .
$$

For all $t>0$ and $\alpha \in(0,1)$, we have

$$
F_{h(p), A(p, q)}(k t) \geq \Delta\left(F_{h(p), h\left(x_{n+1}\right)}(k t-\alpha k t), F_{h\left(x_{n+1}\right), A(p, q)}(k \alpha t)\right)
$$

and

$$
F_{h(q), A(q, p)}(k t) \geq \Delta\left(F_{h(q), h\left(y_{n+1}\right)}(k t-\alpha k t), F_{h\left(y_{n+1}\right), A(q, p)}(k \alpha t)\right) .
$$

Then

$$
\min \left\{F_{h(p), A(p, q)}(k t), F_{h(q), A(q, p)}(k t)\right\} \geq \Delta\left(A_{n}, \min \left\{F_{h\left(x_{n+1}\right), A(p, q)}(k \alpha t), F_{h\left(y_{n+1}\right), A(q, p)}(k \alpha t)\right\}\right),
$$

where

$$
A_{n}=\min \left\{F_{h(p), h\left(x_{n+1}\right)}(k t-\alpha k t), F_{h(q), h\left(y_{n+1}\right)}(k t-\alpha k t)\right\} .
$$

Now, using (31), (32) and (5), we have

$$
\begin{aligned}
& F_{h\left(x_{n+1}\right), A(p, q)}(k \alpha t)=F_{A\left(x_{n}, y_{n}\right), A(p, q)}(k \alpha t) \\
\geq & \min \left\{F_{h\left(x_{n}\right), h(p)}(\alpha t), F_{h\left(y_{n}\right), h(q)}(\alpha t), F_{h\left(x_{n}\right), h\left(x_{n+1}\right)}(\alpha t),\right. \\
& \left.F_{h(p), A(p, q)}(\alpha t), F_{h\left(y_{n}\right), h\left(y_{n+1}\right)}(\alpha t), F_{h(q), A(q, p)}(\alpha t)\right\} \\
& :=B_{n}(\alpha t)=B_{n} .
\end{aligned}
$$

Similarly, we get

$$
F_{h\left(y_{n+1}\right), A(q, p)}(k \alpha t) \geq B_{n} .
$$


Combining (35) and (36), we obtain

$$
\min \left\{F_{h\left(x_{n+1}\right), A(p, q)}(k \alpha t), F_{h\left(y_{n+1}\right), A(q, p)}(k \alpha t)\right\} \geq B_{n} .
$$

Therefore, from (37) and (33), we have

$$
\min \left\{F_{h(p), A(p, q)}(k t), F_{h(q), A(q, p)}(k t)\right\} \geq \Delta\left(A_{n}, B_{n}\right) .
$$

Now, letting $n \rightarrow+\infty$ in (38), using the continuity of the $T$-norm $\Delta$, (30), (20), (21) and the property $\Delta(1, a)=a$ for all $a \in[0,1]$, we get

$$
\min \left\{F_{h(p), A(p, q)}(k t), F_{h(q), A(q, p)}(k t)\right\} \geq \min \left\{F_{h(p), A(p, q)}(\alpha t), F_{h(q), A(q, p)}(\alpha t)\right\} .
$$

Now, letting $\alpha \rightarrow 1^{-}$in the above inequality, using the left-continuity of $F$ and the monotony of $F$, we get

$$
\begin{aligned}
\min \left\{F_{h(p), A(p, q)}(t), F_{h(q), A(q, p)}(t)\right\} & \geq \min \left\{F_{h(p), A(p, q)}(k t), F_{h(q), A(q, p)}(k t)\right\} \\
& \geq \min \left\{F_{h(p), A(p, q)}(t), F_{h(q), A(q, p)}(t)\right\} .
\end{aligned}
$$

Hence, for all $t>0$, we have

$$
\min \left\{F_{h(p), A(p, q)}(t), F_{h(q), A(q, p)}(t)\right\}=\min \left\{F_{h(p), A(p, q)}(k t), F_{h(q), A(q, p)}(k t)\right\} .
$$

Now, applying Lemma 6, we get

$$
A(p, q)=h(p) \quad \text { and } \quad A(q, p)=h(q),
$$

that is, $(p, q)$ is a coupled coincidence point of $A$ and $h$. This makes end to the proof. $\square$ The following result is an immediate consequence of Theorem 7.

Corollary 8. Let $(X, \leq)$ be a partially ordered set and $(X, F, \Delta)$ be a complete Menger PM-space under a T-norm $\Delta$ of H-type. Let $A: X \times X \rightarrow X$ be mapping satisfying the mixed monotone property, for which there exists $k \in(0,1)$ such that

$$
F_{A(x, y), A(u, v)}(k t) \geq \min \left\{F_{x, u}(t), F_{\gamma, v}(t), F_{x, A(x, y)}(t), F_{u, A(u, v)}(t), F_{\gamma, A(\gamma, x)}(t), F_{v, A(v, u)}(t)\right\}
$$

for all $x, y \in X$ for which $x \leq u$ and $y \geq v$ and all $t>0$. Suppose also that

$$
\text { if }\left\{x_{n}\right\} \subset X \text { is a non - decreasing sequence with } x_{n} \rightarrow z \text { in } X \text { then } x_{n} \leq z \text { for all } n \text { hold, }
$$$$
\text { if }\left\{x_{n}\right\} \subset X \text { is a non - increasing sequence with } x_{n} \rightarrow z \text { in } X \text { then } z \leq x_{n} \text { for all } n \text { hold. }
$$

If there exist $x_{0}, y_{0} \in X$ such that

$$
x_{0} \leq A\left(x_{0}, y_{0}\right) \text { and } y_{0} \geq A\left(y_{0}, x_{0}\right),
$$

then $A$ has a coupled fixed point, that is, there exist $p, q \in X$ such that $A(p, q)=p$ and $A(q, p)=q$.

Now, we prove the following result.

Theorem 9. Let $(X, \leq)$ be a partially ordered set and $(X, F, \Delta)$ be a complete Menger PM-space under a T-norm $\Delta$ of H-type. Suppose $A: X \times X \rightarrow X$ and $h: X \rightarrow X$ are two continuous mappings such that $A(X \times X) \subseteq h(X), A$ has the h-mixed monotone property on $X$ and $h$ commutes with $A$. Suppose that for some $k \in(0,1)$,

$$
\begin{aligned}
F_{A(x, y), A(u, v)}(k t) \geq & \min \left\{F_{h(x), h(u)}(t), F_{h(y), h(v)}(t), F_{h(x), A(x, y)}(t),\right. \\
& \left.F_{h(u), A(u, v)}(t), F_{h(y), A(\gamma, x)}(t), F_{h(v), A(v, u)}(t)\right\}
\end{aligned}
$$


for all $x, y \in X$ for which $h(x) \leq h(u)$ and $h(y) \geq h(v)$ and all $t>0$. If there exist $x_{0}, y_{0} \in$ $X$ such that

$$
h\left(x_{0}\right) \leq A\left(x_{0}, y_{0}\right) \text { and } h\left(y_{0}\right) \geq A\left(y_{0}, x_{0}\right),
$$

then $A$ and $h$ have a coupled coincidence point.

Proof. Following the proof of Theorem 7, $\left\{h\left(x_{n}\right)\right\}$ and $\left\{h\left(y_{n}\right)\right\}$ are Cauchy sequences in the complete Menger PM-space $(X, F, \Delta)$. Then, there is some $p, q \in X$ such that

$$
\lim _{n \rightarrow \infty} h\left(x_{n}\right)=p \text { and } \quad \lim _{n \rightarrow \infty} h\left(y_{n}\right)=q .
$$

Since $h$ is continuous, we have

$$
\lim _{n \rightarrow \infty} h\left(h\left(x_{n}\right)\right)=h(p) \quad \text { and } \quad \lim _{n \rightarrow \infty} h\left(h\left(y_{n}\right)\right)=h(q) .
$$

From (8) and the commutativity of $A$ and $h$, we have

$$
h\left(h\left(x_{n+1}\right)\right)=h\left(A\left(x_{n}, y_{n}\right)\right)=A\left(h\left(x_{n}\right), h\left(y_{n}\right)\right)
$$

and

$$
h\left(h\left(y_{n+1}\right)\right)=h\left(A\left(y_{n}, x_{n}\right)\right)=A\left(h\left(y_{n}\right), h\left(x_{n}\right)\right) .
$$

We now show that $h(p)=A(p, q)$ and $h(q)=A(q, p)$. Taking the limit as $n \rightarrow+\infty$ in (41) and (42), by (39), (40) and the continuity of $A$, we get

$$
h(p)=\lim _{n \rightarrow \infty} h\left(h\left(x_{n+1}\right)\right)=\lim _{n \rightarrow \infty} A\left(h\left(x_{n}\right), h\left(y_{n}\right)\right)=A\left(\lim _{n \rightarrow \infty} h\left(x_{n}\right), \lim _{n \rightarrow \infty} h\left(y_{n}\right)\right)=A(p, q)
$$

and

$$
h(q)=\lim _{n \rightarrow \infty} h\left(h\left(y_{n+1}\right)\right)=\lim _{n \rightarrow \infty} A\left(h\left(y_{n}\right), h\left(x_{n}\right)\right)=A\left(\lim _{n \rightarrow \infty} h\left(y_{n}\right), \lim _{n \rightarrow \infty} h\left(x_{n}\right)\right)=A(q, p) .
$$

Thus we proved that $h(p)=A(p, q)$ and $h(q)=A(q, p)$, that is, $(p, q)$ is a coupled coincidence point of $A$ and $h$. This makes end to the proof.

The following result is an immediate consequence of Theorem 9.

Corollary 10. Let $(X, \leq)$ be a partially ordered set and $(X, F, \Delta)$ be a complete Menger PM-space under a T-norm $\Delta$ of H-type. Let $A: X \times X \rightarrow X$ be a continuous mapping having the mixed monontone property, for which there exists $k \in(0,1)$ such that

$$
F_{A(x, y), A(u, v)}(k t) \geq \min \left\{F_{x, u}(t), F_{\gamma, v}(t), F_{x, A(x, y)}(t), F_{u, A(u, v)}(t), F_{\gamma, A(\gamma, x)}(t), F_{v, A(v, u)}(t)\right\}
$$

for all $x, y \in X$ for which $x \leq u$ and $y \geq v$ and all $t>0$. If there exist $x_{0}, y_{0} \in X$ such that

$$
x_{0} \leq A\left(x_{0}, y_{0}\right) \text { and } y_{0} \geq A\left(y_{0}, x_{0}\right) \text {, }
$$

then $A$ has a coupled fixed point.

Now, we end the article with two examples to illustrate our obtained results.

Example 11. Let $(X, d)$ be a metric space defined by $d(x, y)=|x-y|$, where $X=[0,1]$ and $(X, F, \Delta)$ be the induced Menger space with $F_{x, y}(t)=\frac{t}{t+d(x, y)}$ for all $t>0$ and $x, y \in$ $X$. We endow $X$ with the natural ordering of real numbers. Let $h: X \rightarrow X$ be defined as

$$
h(x)=x^{4}, \text { for all } x \in X .
$$

Let $A: X \times X \rightarrow X$ be defined as

$$
A(x, y)=\left\{\begin{array}{lll}
\frac{x^{4}-y^{4}}{4}, & \text { if } & x \geq y \\
0, & \text { if } & x<y .
\end{array}\right.
$$


At first we shall show that the mapping A satisfies the h-mixed monotone property:

Consider $x_{1}, x_{2} \in X$ such that $h\left(x_{1}\right) \leq h\left(x_{2}\right)$. Since $h$ is a non-decreasing mapping, this implies that $x_{1} \leq x_{2}$. Now, let $y \in X$ be an arbitrary point. If $x_{1}<y$, then $A\left(x_{1}, y\right)=0 \leq$ $A\left(x_{2}, y\right)$. If $y \leq x_{1}$, then $\frac{x_{1}^{2}-y^{2}}{4} \leq \frac{x_{2}^{2}-y^{2}}{4}$, that is, $A\left(x_{1}, y\right) \leq A\left(x_{2}, y\right)$. Similarly, one can show that if $y_{1}, y_{2} \in X$ are such that $h\left(y_{1}\right) \geq h\left(y_{2}\right)$, then $A\left(x, y_{1}\right) \leq A\left(x, y_{2}\right)$ for all $x \in$ $X$. Then, the mapping $A$ satisfies the h-mixed monotone property.

Now we shall show that the mappings $A$ and $h$ satisfy the inequality (5). Let $x, y \in X$ such that $h(x) \leq h(u)$ and $h(y) \geq h(v)$ that is, $x^{4} \leq u^{4}$ and $y^{4} \geq v^{4}$.

We have consider the following four cases:

Case-1: $x \geq y$.

Since $x \leq u$ and $y \geq v$, then $u \geq v$. Moreover, for all $t>0$, we have

$$
\begin{aligned}
& F_{A(x, y), A(u, v)}(t / 2)=\frac{t / 2}{t / 2+d(A(x, y), A(u, v))}=\frac{t / 2}{t / 2+\left|\frac{x^{4}-y^{4}}{4}-\frac{u^{4}-v^{4}}{4}\right|} \\
& =\frac{2 t}{2 t+\left|\left(x^{4}-u^{4}\right)-\left(y^{4}-v^{4}\right)\right|} \geq \frac{2 t}{2 t+\left|x^{4}-u^{4}\right|+\left|y^{4}-v^{4}\right|} \\
& \geq \min \left\{\frac{t}{t+\left|x^{4}-u^{4}\right|}, \frac{t}{t+\left|y^{4}-v^{4}\right|}\right\}=\min \left\{F_{h(x), h(u)}(t), F_{h(y), h(v)}(t)\right\} \\
& \geq \min \left\{F_{h(x), h(u)}(t), F_{h(y), h(v)}(t), F_{h(x), A(x, y)}(t), F_{h(u), A(u, v)}(t), F_{h(y), A(\gamma, x)}(t), F_{h(v), A(v, u)}(t)\right\} .
\end{aligned}
$$

Case-2: $x<y$ and $u \geq v$.

In this case, for all $t>0$, we have

$$
\begin{aligned}
& F_{A(x, y), A(u, v)}(t / 2)=\frac{t / 2}{t / 2+d(A(x, y), A(u, v))}=\frac{t / 2}{t / 2+\left|0-\frac{u^{4}-v^{4}}{4}\right|} \\
& =\frac{2 t}{2 t+\left(x^{4}-v^{4}\right)+\left(u^{4}-x^{4}\right)} \geq \frac{2 t}{2 t+\left(y^{4}-v^{4}\right)+\left(u^{4}-x^{4}\right)} \\
& =\frac{2 t}{2 t+\left(u^{4}-x^{4}\right)+\left(y^{4}-v^{4}\right)} \\
& \geq \min \left\{\frac{t}{t+\left(u^{4}-x^{4}\right)}, \frac{t}{t+\left(\gamma^{4}-v^{4}\right)}\right\}=\min \left\{F_{h(x), h(u)}(t), F_{h(y), h(v)}(t)\right\} . \\
& \geq \min \left\{F_{h(x), h(u)}(t), F_{h(y), h(v)}(t), F_{h(x), A(x, y)}(t), F_{h(u), A(u, v)}(t), F_{h(y), A(\gamma, x)}(t), F_{h(v), A(v, u)}(t)\right\}
\end{aligned}
$$

Case-3: $x<y$ and $u<v$.

In this case, for all $t>0$, we have

$$
F_{A(x, y), A(u, v)}(t / 2)=\frac{t / 2}{t / 2+d(A(x, y), A(u, v))}=1 \geq \min \left\{F_{h(x), h(u)}(t), F_{h(y), h(v)}(t)\right\} .
$$

Therefore, the mappings $A$ and $h$ satisfy the inequality (5), as well as all the required hypotheses by Theorem 7 , and $(0,0)$ is the coupled coincidence point of $A$ and $h$.

Example 12. Consider $X=[0,+\infty)$ with

$$
F_{p, q}(t)=\left\{\begin{array}{lll}
\frac{t}{t+\max \{p, q\}} & \text { if } & p \neq q \\
1 & \text { if } & p=q
\end{array}\right.
$$

for all $t>0$ and $p, q \in X$. Then, $\left(X, F, \Delta_{M}\right)$ is a complete Menger PM-space, where $\Delta_{M}(a, b)=\min (a, b)$ for all $a, b \in[0,1]$. We endow $X$ with the natural ordering of real numbers. Define the continuous mapping A: $X \times X \rightarrow X$ by

$$
A(x, y)=\frac{x}{2(1+y)}, \quad \text { for all } x, y \in X \text {. }
$$


Clearly A satisfies the mixed monotone property.

Now we shall show that the mappings $A$ and $h$ satisfy the inequality (5) with $h(x)=x$ for all $x \in X$. Let $(x, y),(u, v) \in X \times X$ such that $x \leq u, y \geq v$ and $A(x, y) \neq A(u, v)$. For all $t>0$, we have

$$
\begin{aligned}
& F_{A(x, y), A(u, v)}(t / 2)=\frac{t}{t+\max \left\{\frac{x}{1+y^{\prime}}, \frac{u}{1+v}\right\}}=\frac{t}{t+\frac{u}{1+v}} \geq \frac{t}{t+u} \geq \frac{t}{t+\max \left\{u, \frac{u}{2(1+v)}\right\}} \\
& =F_{u, A(u, v)}(t) \geq \min \left\{F_{x, u}(t), F_{y, v}(t), F_{x, A(x, y)}(t), F_{u, A}(u, v)(t), F_{y, A(\gamma, x)}(t), F_{v, A(v, u)}(t)\right\} .
\end{aligned}
$$

Therefore, the mappings $A$ and $h$ satisfy the inequality (5), as well as all the required hypotheses by Theorem 9, and $(0,0)$ is the coupled coincidence point of $A$ and $h$, that is, $0=A(0,0)$.

Now, we endow $X$ with the standard metric $d$ given by $d(x, y)=|x-y|$ for all $x, y \in$ $X$. We have

$$
d(A(12,2), A(12,1))=1 \text { and } \frac{d(12,12)+d(2,1)}{2}=\frac{1}{2} .
$$

Then, we cannot find $k \in[0,1)$ such that

$$
d(A(12,2), A(12,1)) \leq \frac{k}{2}[d(12,12)+d(2,1)] .
$$

Then, Theorem 2.1 and Theorem 2.2 of Bhaskar and Lakshmikantham [4]are not applicable in this case. Similarly, we cannot find a function $\phi:[0,+\infty) \rightarrow[0,+\infty)$ with $\phi(t)<t$ for all $t>0$ such that

$$
d(A(12,2), A(12,1)) \leq \varphi\left(\frac{d(12,12)+d(2,1)}{2}\right) .
$$

Then, Theorem 2.1 of Lakshmikantham and Cirić [10]is also not applicable in this case.

\section{Acknowledgements}

The first author is thankful to the Ministry of Science and Technological Development of Serbia.

\section{Author details}

${ }^{1}$ Faculty of Mechanical Engineering, Kraljice Marije 16, 11000 Belgrade, Serbia ${ }^{2}$ Department of Mathematical Sciences, Florida Institute of Technology, Melbourne, FL 32901, USA ³Département de Mathématiques, Université de Tunis, Ecole Supérieure des Sciences et Techniques de Tunis, 5, avenue Taha Hussein-Tunis, B.P.56, Bab Menara-1008, Tunisie

\section{Authors' contributions}

All authors contributed equally and significantly in writing this paper. All authors read and approved the final manuscript.

\section{Competing interests}

The authors declare that they have no competing interests.

Received: 4 March 2011 Accepted: 23 September 2011 Published: 23 September 2011

References

1. Banach, S: Sur les opérations dans les ensembles abstraits et leur application aux équations intégrales. Fund Math. 3, 133-181 (2008)

2. Agarwal, RP, El-Gebeily, MA, O'Regan, D: Generalized contractions in partially ordered metric spaces. Appl Anal. 87, 1-8 (2008). doi:10.1080/00036810701714164

3. Ran, ACM, Reurings, MCB: A fixed point theorem in partially ordered sets and some applications to matrix equations. Proc Am Math Soc. 132, 1435-1443 (2004). doi:10.1090/S0002-9939-03-07220-4

4. Gnana Bhaskar, T, Lakshmikantham, V: Fixed point theorems in partially ordered metric spaces and applications. Nonlinear Anal. 65, 1379-1393 (2006). doi:10.1016/j.na.2005.10.017 
5. Gnana Bhaskar, T, Lakshmikantham, V, Vasundhara Devi, J: Monotone iterative technique for functional differential equations with retardation and anticipation. Nonlinear Anal. 66(10), 2237-2242 (2007). doi:10.1016/..na.2006.03.013

6. Nieto, JJ, López, RR: Contractive mapping theorems in partially ordered sets and applications to ordinary differential equations. Order. 22, 223-239 (2005). doi:10.1007/s11083-005-9018-5

7. Cirić, LB, Lakshmikantham, V: Coupled random fixed point theorems for nonlinear contractions in partially ordered metric spaces. Stoch Anal Appl. 27(6), 1246-1259 (2009). doi:10.1080/07362990903259967

8. Cirić, LB, Miheț, D, Saadati, R: Monotone generalized contractions in partially ordered probabilistic metric spaces. Topol Appl. 156(17), 2838-2844 (2009). doi:10.1016/j.topol.2009.08.029

9. Ćirić, LB, Cakić, N, Rajović, M, Ume, JS: Monotone generalized nonlinear contractions in partially ordered metric spaces. Fixed Point Theory Appl. Article ID 131294 (2008)

10. Lakshmikantham, V, Cirić, LB: Coupled fixed point theorems for nonlinear contractions in partially ordered metric spaces. Nonlinear Anal. 70(12), 4341-4349 (2009). doi:10.1016/j.na.2008.09.020

11. Nieto, JJ, López, RR: Existence and uniqueness of fixed point in partially ordered sets and applications to ordinary differential equations. Acta Math Sinica, English Series. 23, 2205-2212 (2007). doi:10.1007/s10114-005-0769-0

12. Samet, B: Coupled fixed point theorems for a generalized Meir-Keeler contraction in partially ordered metric spaces. Nonlinear Anal. 72(12), 4508-4517 (2010). doi:10.1016/j.na.2010.02.026

13. Samet, B, Vetro, C: Coupled fixed point theorems for multi-valued nonlinear contraction mappings in partially ordered metric spaces. Nonlinear Anal. 74, 4260-4268 (2011). doi:10.1016/j.na.2011.04.007

14. Samet, B, Yazidi, H: Coupled Fixed point theorems in partially ordered $\varepsilon$-chainable metric spaces. J Math Comput Sci. $1(3), 142-151$ (2010)

15. Menger, K: Statistical metric. Proc Natl Acad USA. 28, 535-537 (1942). doi:10.1073/pnas.28.12.535

16. Schweizer, B, Sklar, A: Statistical metric spaces. Pacific J Math. 10, 313-334 (1960)

17. Schweizer, B, Sklar, A: Probabilistic Metric Spaces. Elsevier North Holand, New York (1983)

18. Sehgal, VM, Bharucha-Reid, AT: Fixed points of contraction mappings on probabilistic metric spaces. Math Syst Theory. 6, 97-102 (1972). doi:10.1007/BF01706080

19. Cirić, LB: Some new results for Banach contractions and Edelstein contractive mappings on fuzzy metric spaces. Chaos Solitons Fractals. 42, 146-154 (2009). doi:10.1016/j.chaos.2008.11.010

20. Cirić, LB, Ješić, SN, Ume, JS: The existence theorems for fixed and periodic points of nonexpansive mappings in intuitionistic fuzzy metric spaces. Chaos Solitons Fractals. 37(3), 781-791 (2008). doi:10.1016/j.chaos.2006.09.093

21. Fang, JX, Gao, Y: Common fixed point theorems under strict contractive conditions in Menger spaces. Nonlinear Anal. 70, 184-193 (2009). doi:10.1016/j.na.2007.11.045

22. Hadžić, O, Pap, E: Fixed Point Theory in PM Spaces. Kluwer, Boston (2001)

23. Khamsi, MA, Kreinovich, VY: Fixed point theorems for dissipative mappings in complete probabilistic metric spaces. Math Jap. 44, 513-520 (1996)

24. Miheț, D: A generalization of a contraction principle in probabilistic metric spaces (II). Int J Math Math Sci. 5, 729-736 (2005)

25. Miheț, D: Fixed point theorems in probabilistic metric spaces. Chaos Solitons Fractals. 41(2), 1014-1019 (2009). doi:10.1016/j.chaos.2008.04.030

26. Miheț, D: Altering distances in probabilistic Menger spaces. Nonlinear Anal. 71, 2734-2738 (2009). doi:10.1016/j. na.2009.01.107

27. O'Regan, D, Saadati, R: Nonlinear contraction theorems in probabilistic spaces. Appl Math Comput. 195, 86-93 (2008). doi:10.1016/j.amc.2007.04.070

28. Cirić, LB: A generalization of Banach's contraction principle. Proc Am Math Soc. 45, 267-273 (1974)

29. Ćirić, LB: Coincidence and fixed points for maps on topological spaces. Topol Appl. 154, 3100-3106 (2007). doi:10.1016/ j.topol.2007.08.004

30. Hussain, N: Common fixed points in best approximation for Banach operator pairs with Ćirić Type I-contractions. J Math Anal Appl. 338, 1351-1363 (2008). doi:10.1016/j.jmaa.2007.06.008

31. Liu, Z, Guo, Z, Kang, SM, Lee, SK: On Ćirić type mappings with nonunique fixed and periodic points. Int J Pure Appl Math. 26(3), 399-408 (2006)

32. Lakshmikantham, V, Mohapatra, RN: Theory of Fuzzy Differential Equations and Inclusions. Taylor\&Francis, London. (2003)

33. Lakshmikantham, V, Koksal, S: Monotone flows and rapid convergence for nonlinear partial differential equations. Taylor\&Francis. (2003)

34. Lakshmikantham, V, Vatsala, AS: General uniqueness and monotone iterative technique for fractional differential equations. Appl Math Lett. 21(8), 828-834 (2008). doi:10.1016/j.aml.2007.09.006

35. Nashine, HK, Samet, B: Fixed point results for mappings satisfying $(\psi, \varphi)$ weakly contractive condition in partially ordered metric spaces. Nonlinear Anal. 74, 2201-2209 (2011). doi:10.1016/j.na.2010.11.024

36. Samet, B: Ćirić's fixed point theorem in a cone metric space. J Nonlinear Sci Appl. 3(4), 302-308 (2010)

37. Singh, SL, Mishra, SN: On a Ljubomir Ćirić's fixed point theorem for nonexpansive type maps with applications. Indian J Pure Appl Math. 33, 531-542 (2002)

doi:10.1186/1687-1812-2011-56

Cite this article as: Ćirić et al: Mixed monotone-generalized contractions in partially ordered probabilistic metric spaces. Fixed Point Theory and Applications 2011 2011:56. 